\title{
SURGICAL TREATMENT FOR DERANGEMENT OF THE LUMBAR SPINE
}

\author{
P. H. Newman, London, England
}

Before the details of operative treatment for mechanical derangement of the lumbar spine are considered it is important to have some idea of the part that surgical intervention plays in the broad picture of backache and sciatica. The answer must inevitably be that it plays only a very small part. Only a minority of people suffering from these complaints seek advice from their general practitioner; the majority cope with the trouble themselves and some seek aid from the many other non-medically qualified experts in the differing media available to such sufferers.

More accurate figures show that of 100 patients reporting to their general practitioner, ten are referred to hospital and less than one is treated by operation. Approximately one person in a population of 10,000 is treated surgically each year for spinal derangement.

Terminology-There is some confusion in the nomenclature used for the different operative procedures on the low back.

Laminectomy can be correctly used to describe the excision of a lamina or the excision of two or more laminae. It can indicate the excision of the neural arch on one side of the midline between the spinous process and pedicle or excision of the whole neural arch between the two pedicles (two laminae) or excision of multiple neural arches. Unfortunately the term laminectomy is often used loosely as a general term to describe almost any operation on the lumbar spine including fenestration, stabilisation or facetectomy.

Hemilaminectomy is sometimes used to describe a single laminectomy or laminotomy. It has no place in operative terminology.

Fenestration is an objectively descriptive but not an anatomically accurate term. It indicates exploration of the spinal canal and lateral recess through an inspection aperture. It necessitates removal of ligamentum flavum and possibly the adjoining edge of the lamina proximally and distally and occasionally the medial border of the facets, avoiding severance of the neural arch and with minimal disturbance of the zygapophysial joint.

Lumbar spondylotomy is a useful comprehensive term to include all surgical interventions that involve cutting into the vertebral column, whether soft-tissue or bone; it can therefore rightfully include all operations opening the spinal or intervertebral canals or simple procedures for stabilisation of two or more vertebrae.

Rhachiotomy (rachiotomy) has a similar meaning but is used much less often.

\section{INDICATIONS FOR SURGICAL TREATMENT}

Some clinical syndromes present more clearly than others and the indications for surgical treatment evolve when some definite combination of circumstances arises. The severity of symptoms, the lack of response to simple therapeutic measures, confidence as to the true origin of the pain, the passage of time and the modification of activity are factors influencing the decision. In addition the patient may find himself unwilling or incapable of coping with his symptoms and positively seeks a more active and definite means of treatment. The greater the severity of the pain and the greater the interference with function, the less will the time factor assume significance.

It is those syndromes which arise from a recognisable morbid pathological lesion which tend to develop into persistent trouble and may be amenable to surgical treatment. Operation should be contemplated only when the reason for intervention is clear: there is very little place for an exploratory operation on the spinal canal, unless of course infective or neoplastic disease is suspected. 


\section{SURGICAL OBJECTIVE}

Surgical intervention for derangement of the spine has three main objectives: 1) removal of a space-occupying lesion; 2) stabilisation; and 3) decompression. A correct diagnosis of the real cause of the symptoms is aided by accurate knowledge of the morbid pathology of this area and of the clinical syndromes commonly associated with each type of lesion. Careful clinical examination supported by diagnostic aids and investigations will help to eliminate a false diagnosis and hence a badly designed operation. So often in assessment of musculoskeletal derangements the clinical history provides the most valuable evidence in the surgeon's endeavour to decide the correct treatment for each case. The low back is no exception. Simple radiographic changes such as intervertebral space diminution, spondylolysis or spondylolisthesis, and myelographic and discographic changes may occasionally detract from the diagnosis of the real cause of the symptoms. The interpretation of the true origin of the pain is the key to a well planned operation.

\section{SYNDROMES RESPONSIVE TO SURGERY}

From among the complexity and confusion of the syndromes arising from derangement of the lumbar spine a few emerge with a more definite clinical picture, a clearer morbid anatomical cause and a more ready response to surgery than others. Eight have been selected as the most suitable for brief description, including those that are the most common and those that present the best prospect of relief by operation: 1) lateral disc prolapse; 2) central disc prolapse; 3) instability; 4) congenital spondylolisthesis; 5) spondylolytic spondylolisthesis; 6) degenerative spondylolisthesis; 7) spinal stenosis, primary or secondary; 8) lumbo-sacral disc degeneration and nerve root entrapment.

\section{LATERAL DISC PROLAPSE}

This syndrome is the most obvious and easily recognisable of those in this group. It is common and one of the most amenable to surgical treatment. It occurs most frequently in men between the ages of twenty and fifty years.

A history of low back pain with intervening acute unilateral sciatica radiating in the distribution of the sciatic nerve and affected nerve, felt particularly at the sciatic notch and at the upper and outer aspect of the leg, is typical. There is marked stiffness of the lumbar spine in flexion and extension but none in lateral flexion. A list to either side may be present and lordosis is often obliterated. Limitation of straight leg raising may be marked, with pain localised to the sciatic notch: similar pain may be elicited by raising the opposite leg or by flexing the neck. There may be neurological evidence of alteration in nerve conduction, such as diminution or absence of reflexes, alteration in sensation or loss of motor power. This syndrome can be so clear that further investigation apart from simple radiographic examination is unnecessary. Should doubt prevail, examination of the cerebrospinal fluid and myelography may help to eliminate the presence of a more unusual type of lesion.

The operative findings in two individual reports, each of 500 cases with lumbar disc prolapse, show very similar figures (O’Connell 1951, Robinson 1965) (Table I).

\section{OPERATIVE TECHNIQUE}

A lateral prolapse of an intervertebral disc can be approached by an interlaminar exposure, commonly known as a fenestration operation.

The patient can be positioned kneeling, lying prone or, as in our clinic, in the lateral posture with the knees and hips flexed and the table broken convex into the loin. The incision separates the sacrospinalis from one side of the spinous processes, leaving the median structures intact. Exposure is completed by removal of the ligamentum flavum and if necessary the 
adjoining edges of the lamina above and below and the median edges of the facets laterally. The wider the exposure the easier, in principle, to remove the prolapsed disc substance; but the art of surgery for derangement of the lumbar spine is to obtain adequate exposure with minimal interference with intersegmental control.

The operation over, it is psychologically important to regard the procedure as a comparatively minor one. Walking in a few days and flexion and extension movement of the spine after ten days are important for two reasons: to mobilise the nerve tissues and to build up the intrinsic intersegmental muscles. Flexion accounts for the former, recovery by extension for the latter.

TABLE I

Clinical Details in Two Series of Patients with lateral Disc Prolapse

\begin{tabular}{|c|c|c|c|c|c|}
\hline \multirow{2}{*}{\multicolumn{2}{|c|}{$\frac{\text { Author } .}{\begin{array}{c}\text { Age } \\
(\text { years })\end{array}}$}} & \multicolumn{2}{|c|}{ O'Connell (1951) } & \multicolumn{2}{|c|}{ Robinson (1965) } \\
\hline & & $\begin{array}{l}\text { Number of } \\
\text { patients }\end{array}$ & Per cent & $\begin{array}{l}\text { Number of } \\
\text { patients }\end{array}$ & Per cent \\
\hline $11-20$ & . & 20 & 4 & 17 & $3 \cdot 5$ \\
\hline $21-30$ & . & 174 & 35 & 102 & 20 \\
\hline $31-40$. & . & 190 & 38 & 190 & 38 \\
\hline $41-50$ & . & 93 & $18 \cdot 5$ & 134 & 27 \\
\hline $51-60$ & . & 23 & $4 \cdot 5$ & 42 & $8 \cdot 5$ \\
\hline \multicolumn{6}{|c|}{ Sex } \\
\hline Males & . & 330 & 66 & 347 & 69 \\
\hline Females & 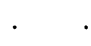 & 170 & 34 & 153 & 31 \\
\hline \multicolumn{6}{|c|}{ Site } \\
\hline Lumbar 2 & ad 3 & 8 & $1 \cdot 6$ & 17 & $3 \cdot 4$ \\
\hline Lumbar 4 & ${ }^{\circ}$ & 198 & $39 \cdot 6$ & 189 & $37 \cdot 8$ \\
\hline Lumbar 5 & . & 248 & $49 \cdot 6$ & 294 & $58 \cdot 8$ \\
\hline (Double pr & trusion) & 46 & $9 \cdot 2$ & & \\
\hline
\end{tabular}

\section{CHEMONUCLEOLYSIS}

The treatment of prolapsed intervertebral disc by the injection of chymopapain, a proteolytic enzyme, is an alternative method and has now been on trial for nine years.

Recently Dr Lyman Smith (1972) reported the combined experiences of twenty-three investigators. The results in a total of 2,486 patients were encouraging. However, the injection needs a general anaesthetic and fluoscopic control for needle localisation. Anaphylactic shock occurs in about 1 per cent of cases and there is a danger of ventricular fibrillation if adrenalin is given under fluothane anaesthesia.

\section{CENTRAL DISC PROLAPSE}

Central disc prolapse is associated with loss of integrity of the posterior longitudinal ligament. Massive prolapse can produce a very significant syndrome of intense pain, spasm of the lower spinal and abdominal muscles and evidence of compression of the cauda equina: but at the other extreme quite marked prolapse can be accommodated in the spinal canal with minimal symptoms.

VOl. 55 B, NO. 1, FEBRUARY 1973 
Central prolapse is much less common than lateral prolapse. It is more frequent in men (70 per cent), with a higher age range, thirty to seventy years, and a higher incidence at the lumbar 3-4 and 4-5 levels.

Recurrent or chronic back pain of long duration, sometimes with mild unilateral sciatica, changes to an acute syndrome of bilateral sciatica, weakness of legs, paraesthesiae in the lower legs and feet, hypoaesthesia over buttocks and back of thighs and paralysis of the bladder. In the event of obvious evidence of compression of the cauda equina, including in particular the last two factors mentioned, the case must be regarded as a potential surgical emergency, and if bladder function does not respond rapidly to bed rest, exploration should be undertaken forthwith, because abrupt compression of nerve tissue is so much more prone to cause permanent damage than slowly increasing pressure as occurs in neoplastic disease. The true nature of the lesion may be much more difficult to diagnose when symptoms are less severe and signs less obvious. Chronic backache in some cases may be the only symptom in a surprisingly severe degree of central disc prolapse, and limitation of straight-leg raising is typically not so prominent a sign as it is in lateral disc prolapse. A raised protein level in the cerebrospinal fluid is common, and myelography will reveal the level and the severity of the prolapse in addition to eliminating a spinal neoplasm.

\section{OPERATIVE TECHNIQUE}

The overruling priority in massive central prolapse is to relieve compression of the cauda equina. Wide exposure by laminectomy of one or more vertebrae is essential for adequate exposure and removal of disc substance with minimal trauma to nerve tissues.

The patient lies prone on a support which eliminates pressure on the abdomen and permits free respiration. The sacrospinalis is retracted on both sides, giving full exposure of the neural arches and the appropriate spinous process, and laminae are removed from one or more vertebrae. In massive central prolapse the cauda equina is liable to be displaced posteriorly hard against the neural arch, and care is needed not to injure it by placing forceps anterior to the laminae. The prolapsed tissue can be removed either by reflecting the theca or transdurally, a decision which must be made when the theca is exposed.

\section{DISC REMOVAL AND STABILISATION}

So far two distinct operations have been described, fenestration for persistent lateral disc prolapse and decompression and removal of prolapsed disc tissue for massive central prolapse. There is a third group which is common but less well defined, and the indications for operation and the technique of operation are more controversial. It is those cases with chronic persistent backache or recurrent attacks of acute low back pain, with obvious or less apparent nerve involvement, which present difficulty both in diagnosis and in treatment. The surgical details must of course be planned on individual evidence. Typically these patients are of middle age. Exploration of the lower two spaces may be considered necessary according to the presence in the history of unilateral or bilateral sciatica. The question of stabilisation will also arise. There are some who consider that stabilisation is never indicated at the time of disc removal, whereas others advocate fusion when there is evidence of instability, chronic severe disc degeneration and osteoarthritis of the zygapophysial joints. This point was discussed by Connolly and Newman (1971).

\section{INSTABILITY}

The symptom of instability is essentially low back pain, but this has many variations. Instability can be defined as a loss of integrity of soft-tissue intersegmental control causing potential weakness and liability to yield under stress. The lesion may be activated by compression, by flexion, by extension or by rotatory stress. Recurrent attacks of acute low 
back pain are sometimes heralded by a feeling of "tearing", "giving way" or "something going out of place" in the back. The patient may be fixed in a flexed position, leaning on the furniture for support. Complete collapse to the floor indicates a combination of mechanical failure and central prolapse. These patients complain of constant back discomfort possibly arising from muscle fatigue or neural arch stress in maintaining a protective extension posture. The most common level for instability is between the fourth and fifth lumbar vertebrae, and pain from this site characteristically radiates across the lower back, round the buttocks, into the groins.

The diagnosis of instability rests largely on the detailed history, for clinical signs are not abundant. Spasm in the sacrospinalis muscle holding an extension posture of the lumbar spine, midline tenderness and deficiency of the supraspinous raphe on palpation are suggestive. Radiographic evidence is unreliable. Subluxation posteriorly, diminution of disc space and an abnormal range of rocking in flexion and extension are significant. Discography can be helpful, especially in the mid-lumbar spine, if only one disc is found abnormal and if the pain produced at the time of injection is similar to the complaint.

\section{OPERATIVE TECHNIQUE}

The method of stabilisation selected in our clinic depends upon the integrity of the neural arch. When the neural arch and articular facets are intact the technique of Boucher (1959) with long transarticular screws and posterior laminar arthrodesis is used. This method is unsatisfactory when the neural arch is not intact, as for instance in spondylolisthesis or when laminectomy has been previously performed or when there is severe spina bifida occulta.

Fusion with intact neural arch-The screw fusion as described by Boucher has been found successful in a high proportion of patients, with solid mechanical fusion in at least 95 per cent of cases. It cannot be claimed that relief of symptoms occurs in the same proportion, but it is not considered that failure to relieve symptoms is often caused by pseudarthrosis. Faulty assessment of the true cause of pain, or of the level of the lesion, or operative damage to softtissues of the segment above the fusion, which causes potential weakness, are probably the more likely causes of failure. In planning arthrodesis the levels to be stabilised must be clearly defined because avoidance of damage to segments above or below is essential.

The long-term results of screw fusion have given some concern, for hypertrophy of the neural arches causing secondary stenosis has been known to occur. It is possible that posterolateral as opposed to supralaminar fusion will become the method of choice in the future but its long-term results may also reveal defective factors.

Fusion with defective neural arch-Provided a wide exploration of the spinal canal is not contemplated, the lateral gutter can best be approached through the substance of the sacrospinalis muscle. The incision described by Wiltse, Bateman, Hutchinson and Nelson (1968) has been found very practical and simple (see page 14). Roots can be decompressed in the lateral spinal recesses or in the intervertebral canals by lateral laminectomy or facetectomy. The outer aspect of the ilium can be readily exposed through either incision as a donor site for bone cortex and medullary slivers. The transverse processes, the outer aspect of the facets and the adjoining laminae are decorticated and each gutter is packed with bone slivers.

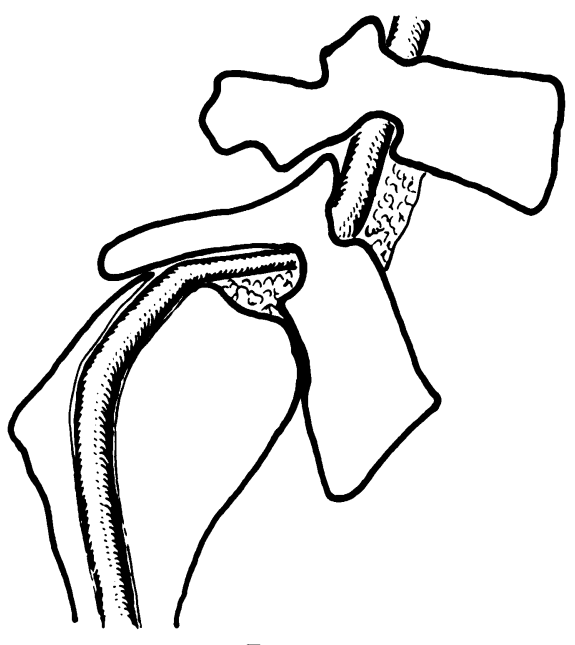

FIG. 1

A diagram to demonstrate the cause of tension on the cauda equina in cases of congenital
spondylolisthesis. 


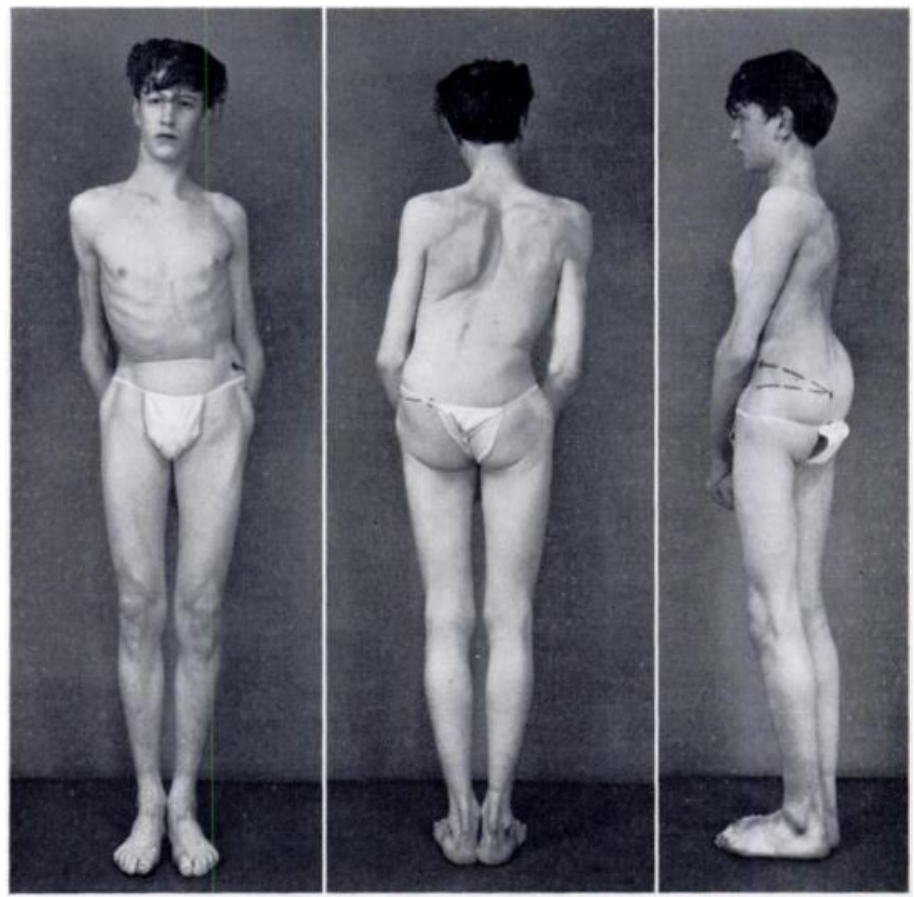

Fig. 2

Case 1-Typical spinal deformity of congenital spondylolisthesis.

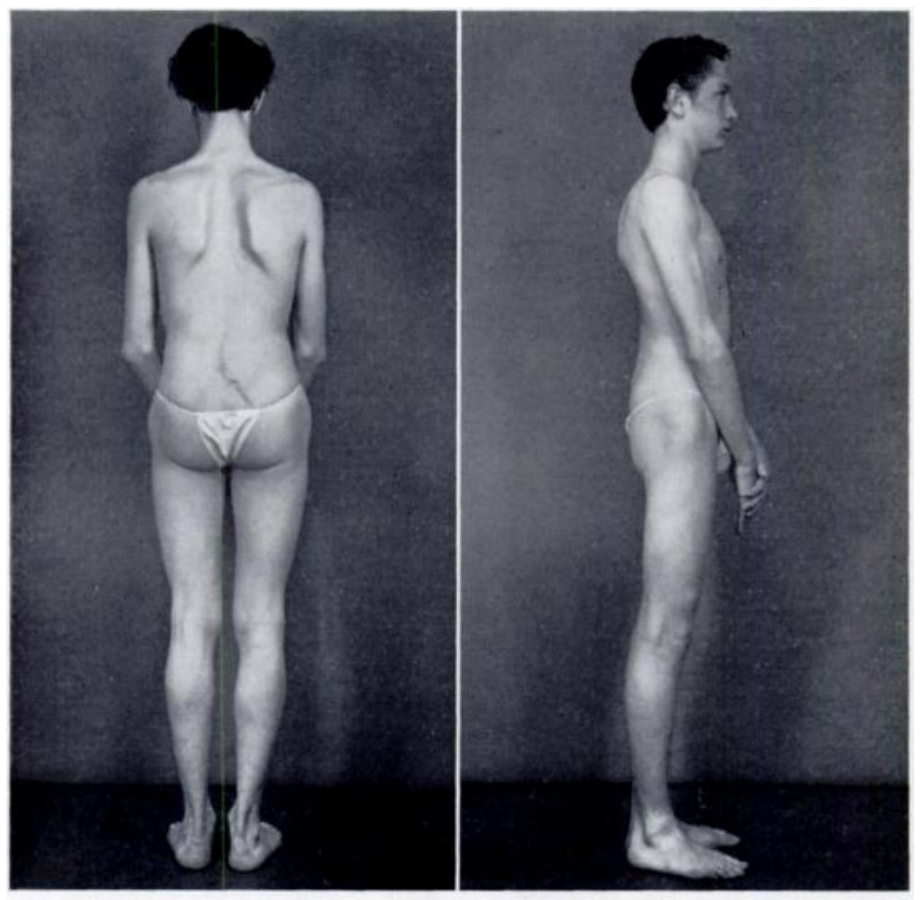

FIG. 3

Case 1-The same patient one year after postero-lateral fusion. 


\section{CONGENITAL SPONDYLOLISTHESIS}

In this type of spondylolisthesis the congenital factor is the absence of proper development of the upper part of the sacrum which permits lumbo-sacral luxation. Spondylolisthesis is not present at birth but the deficiency of the sacral facets and the sacral neural arches is a potential weakness to the constant forward thrust. Lumbo-sacral subluxation progresses slowly but tends to advance more rapidly during the adolescent spurt of growth. This is almost invariably when symptoms are noticed and medical advice is sought; between twelve and fourteen years in girls and between fourteen and sixteen in boys are the peak periods. It is three times more common in girls.

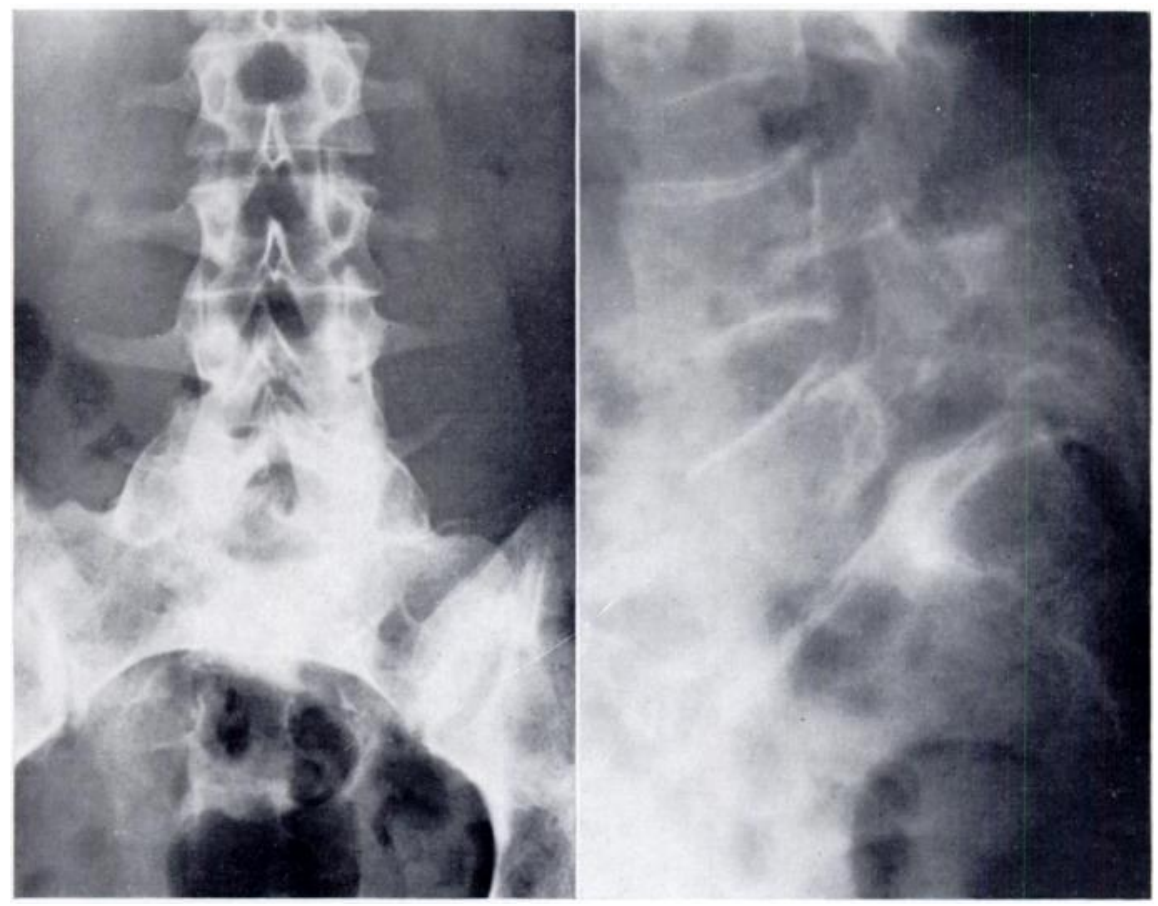

FIG. 4

Case 1-The postero-lateral fusion. There has been no decompression.

The patient complains of stiffness and of discomfort in the thighs and legs sometimes amounting to pain. Heaviness of the legs and paraesthesiae in the feet are common: objective sensory loss and altered bladder function are less common. Retention of urine may occur.

Examination reveals a shortened trunk compared to the length of the lower limbs, a prominent sacrum with increased lordosis above, backward tilting of the pelvis, and skin creases in the loin (Fig. 2). Forward flexion is markedly restricted and straight-leg raising is also very limited. Forcing the leg further may lift the buttock from the couch without obvious pain, a sign typical of cauda equina tension in adolescents. Radiographically the fifth lumbar vertebra is seen displaced forward on the sacrum, often more than half a diameter: sometimes there is complete displacement of the last lumbar vertebral body anterior to the sacrum. The syndrome is caused by tension on the cauda equina, stretched between the posterior aspect of the neural arches of the fourth and fifth lumbar vertebrae anteriorly and the posterior aspect of the first sacral body posteriorly (Fig. 1).

Stabilisation is indicated in all cases with a severe degree of luxation with symptoms, in order to prevent further slip, to eliminate symptoms and to prevent increase in deformity (especially scoliosis), and to prevent the bladder paralysis which occasionally complicates the clinical picture. 


\section{OPERATIVE TECHNIQUE}

Stabilisation is the main objective: decompression is necessary only if there is severe or increasing evidence of cauda equina involvement. Although the pars interarticularis may be intact in these cases, the lumbo-sacral joints have given way and the back of the sacrum is defective, so that supralaminar fusion with screw fixation is not possible.

Fusion is achieved with slivers placed in the postero-lateral gutter through bilateral incisions. Grafts are laid over the decorticated transverse processes of the fourth and fifth lumbar vertebrae and the alae of the sacrum, and over the facets and the adjoining lateral halves of the laminae. The incidence of fusion in these adolescents is high and the relief of deformity, stiffness and pain by simple stabilisation remarkably good (Figs. 3 and 4).

\section{SPONDYLOLYTIC SPONDYLOLISTHESIS}

This is the most common type of spondylolisthesis, occurring slightly more frequently in males and found at any age except during the first year after birth. It is associated with spondylolysis, a lesion of the pars interarticularis of the neural arch. The amount of forward slip of the vertebral body may be greater than the distance of separation at the break, indicating that there is a factor additional to simple separation. The degree of displacement is typically only moderate, but it is inclined to be greater when the slip begins in childhood or adolescence; complete forward luxation, as seen in the congenital variety, does not occur.

It is not unusual for this lesion to remain symptomless and for its presence to be discovered in routine lumbar radiography. When symptoms are present they tend to fall into one or more of three categories, and these constitute the characteristic syndrome of the lesion.

There are the symptoms of instability, chronic low postural backache, recurrent lumbago and inability to bend and lift from the flexed position. Uncommon but more characteristic is a pain spreading down the back of both thighs to the knees or slightly lower, which may be due to recurrent traction on the first and second sacral nerves as they pass over the prominent hump of the posterior border of the first sacral vertebral body, accentuated of course by the excessive movement of mechanical instability. The third category is a true sciatica in the distribution of the nerve of the same numerical value as the vertebra involved. The nerve is trapped by the fibrous tissue and callus of the pseudarthrosis as it turns around the pedicle.

Surgical treatment is indicated when symptoms have become a burden to normal function and a hindrance to the enjoyment of normal activity and are uncontrolled by a corset or other simple measures. The operation is planned according to the symptoms. The pain and discomfort of instability and of traction of the first and second sacral nerve roots should be relieved by simple fusion. True sciatica from nerve entrapment will need additional decompression.

\section{OPERATIVE TECHNIQUE}

With a lesion in the neural arch posterior arthrodesis of the laminae with transarticular screws is not satisfactory. Fusion must be achieved either by the postero-lateral intertransverse or by the anterior intercorporeal method. The former is a safer procedure in the hands of the average surgeon and has the additional advantage of permitting decompression when indicated.

There are many ways of approaching the alae of the sacrum and the lower lumbar transverse processes. In all, the approach is made with the patient prone on a pelvic support. The simple model used in our clinic is shown in Figure 5. A choice of incisions is available.

A midline incision retracting both sacrospinalis muscles laterally has the advantage of permitting full decompression or exploration of the spinal canal but the disadvantage of a difficult exposure of the transverse processes in fat or muscular patients. Bilateral curved incisions, concave medially, give an easy exposure of the transverse processes by cutting vertically through the sacrospinalis between its outer and middle thirds. This approach leaves 
the supraspinous ligament, the spinous process and adjoining neural arch intact and does permit decompression of the lateral recess of the spinal canal (Wiltse et al. 1968). A further alternative is to make bilateral incisions over both posterior iliac spines, reflecting the sacrospinalis muscles medially with their iliac attachment. This incision gives good exposure of the lateral gutter but does not permit easy decompression of the lateral recess. The detached bone should be used for reattachment and not for grafting. Finally, the skin incision may be a transverse one, slightly concave proximally, with a transverse incision across the sacrospinalis.

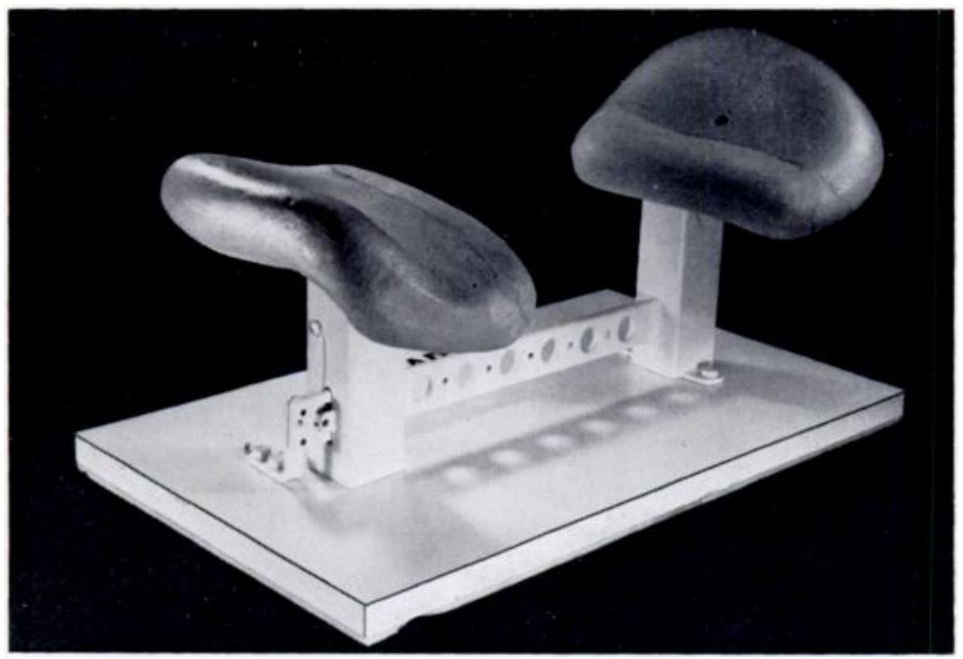

Fic. 5

A simple pelvic support with two resilient plastic cups, adjustable to the width of the pelvis.

This gives a good exposure of the lateral gutter and permits full or partial decompression. Repair of the muscle belly is difficult and tends to leave a void into which bone graft slivers may be displaced.

Decompression of the appropriate nerve, either on one side or both, can easily be achieved by these routes. If the whole posterior neural arch needs to be removed the bilateral approaches are not suitable. Bone can be taken from the outer side of the ilium through any of these approaches, but it is necessary to give a J-shaped extension to the single midline incision.

\section{DEGENERATIVE SPONDYLOLISTHESIS}

Degenerative spondylolisthesis is three times more common in women than in men and is not seen under the age of forty. In the great majority of cases it occurs at the 4-5 lumbar level. Radiologically the fourth lumbar vertebra slips forward, never more than a moderate degree: usually the displacement does not progress further than one-fifth of the vertebral body diameter. The neural arch is intact, but there is subluxation of the zygapophysial joints, which show osteoarthritic degenerative change.

The clinical syndrome has four characteristic components. With some patients a long history, suggestive of chronic instability, may be extracted. It is probable that this degenerative type of spondylolisthesis is a late phenomenon of chronic intersegmental instability.

Osteoarthritis of the zygapophysial joints causes chronic low back discomfort and stiffness after rest, relieved by heat and aggravated by cold. As the hypertrophic arthritic change develops the inferior articular facets of the fourth vertebra grind their way forward between 
16

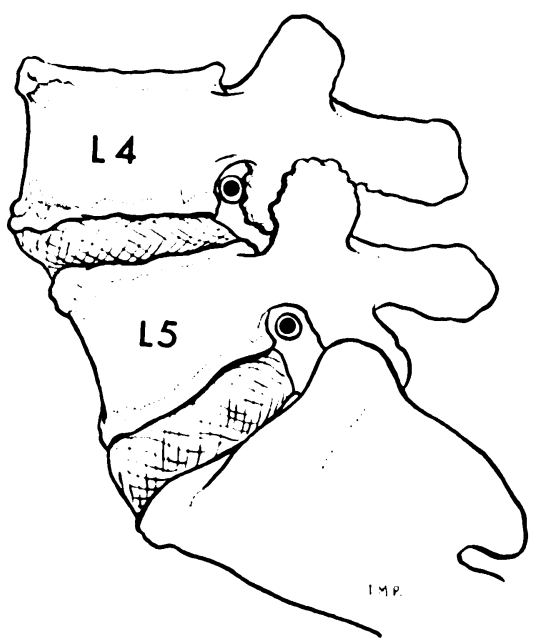

FIG. 6

Degenerative spondylolisthesis.
P. H. NEWMAN

the superior facets of the fifth. The forward subluxation combined with periarticular thickening and osteophytic outgrowth may cause entrapment of the fifth lumbar nerve (Fig. 6). This gives the third component of the syndrome, the onset during a long disability from low backache, of sciatica and possibly loss of sensation and muscle weakness appropriate to this nerve. The fourth component of the clinical picture, which occurs occasionally, is that of spinal stenosis caused by forward subluxation and hypertrophic change, giving the characteristic syndrome from pressure on the theca enclosing the cauda equina, to be described in the next section.

\section{OPERATIVE TECHNIQUE}

Surgical intervention in this type of spondylolisthesis is indicated when fifth lumbar nerve sciatica or symptoms of spinal stenosis do not respond to conserva-

tive measures. The pain caused by osteoarthritic change can often be adequately relieved by a well made surgical corset.

The primary object of operation is to decompress the theca and both fifth lumbar nerves. This must be achieved, no matter how much bone is removed. Partial bilateral laminectomy with partial bilateral vertical facetectomy is generally sufficient, but occasionally the whole neural arch of the fourth lumbar vertebra will have to be removed to give the surgeon ample assurance that decompression is complete. The approach and the decompression in this condition can be quite difficult owing to the marked hypertrophy of the facet edges and the forward subluxation.

Fusion is desirable in order to prevent chronic backache and further forward subluxation. It can be achieved at the same operation by postero-lateral fusion performed by retracting the sacrospinalis muscle laterally and placing bone slivers over the decorticated bony elements of the lateral gutter. Alternatively fusion can be undertaken at a second operation through an exposure of the appropriate vertebral bodies by an extraperitoneal route through a loin incision.

\section{SPINAL STENOSIS}

The term spinal stenosis was probably used originally to describe congenital narrowing of the spinal canal. Sarpyener (1945) reported narrowing of the canal associated with spina bifida occulta. Verbiest $(1954,1955)$ reported congenital narrowing of the lumbar spinal canal and demonstrated the importance of its potentiality in precipitating symptoms

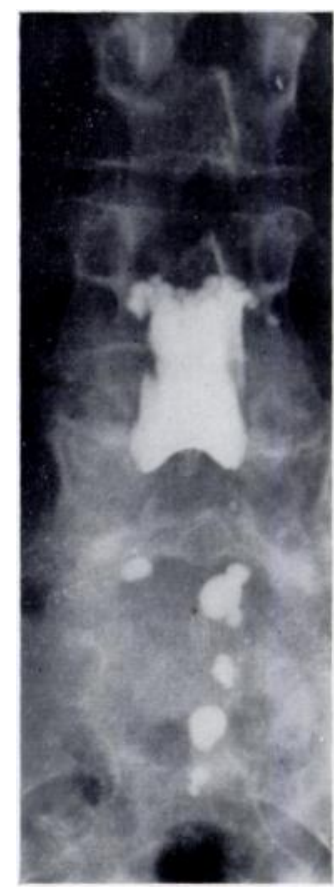

FiG. 7

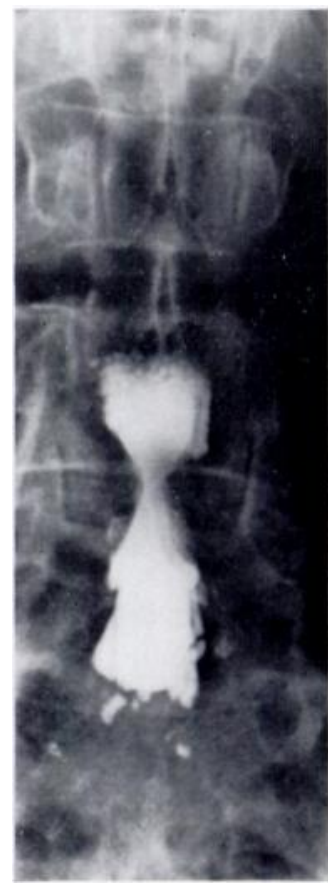

Fig. 8

Myelography in two patients with degenerative spondylolisthesis. Figure 7-Showing complete block. Figure 8-Showing partial block. Note the typical hour-glass appearance caused by encroachment on the spinal canal by an osteoarthritic joint postero-laterally on each side. 
when associated with comparatively minor degrees of disc prolapse (Figs. 9 and 10). The normal lumbar spinal canal tends to be smaller in its antero-posterior measurement at the lumbar 3-4 and 4-5 levels, particularly in men. In women the canal shows a tendency to flattening at the lumbo-sacral level. An abnormal degree of flattening is associated with short pedicles and is found to a marked degree in subjects with achondroplasia, who are prone to develop symptoms of spinal stenosis.

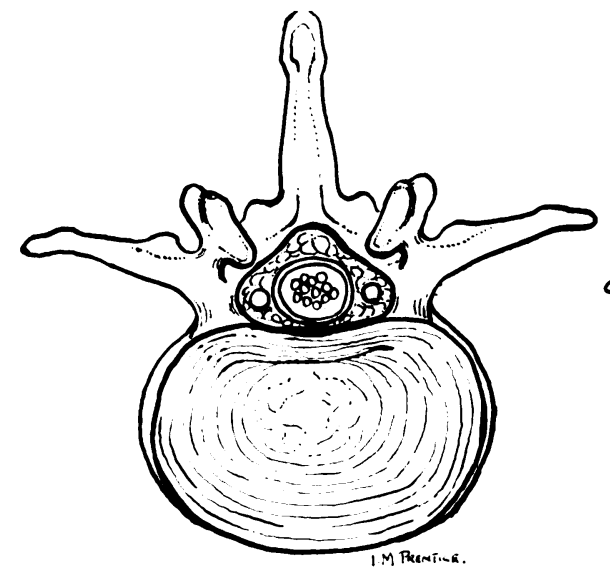

FIG. 9

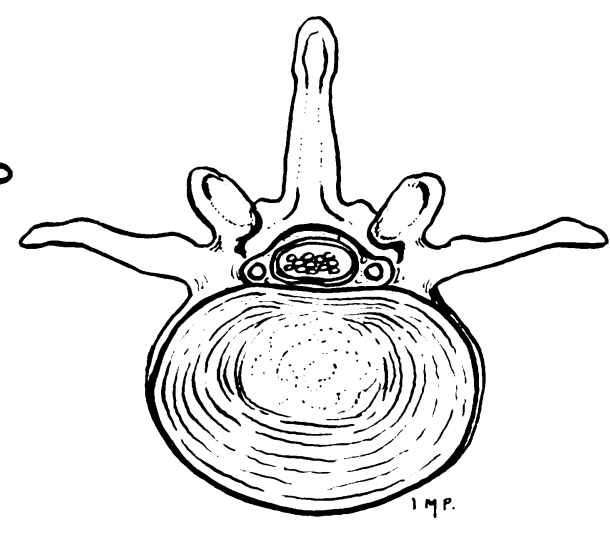

FIG. 10

Figure 9-A normal canal. Figure 10-A flattened canal.

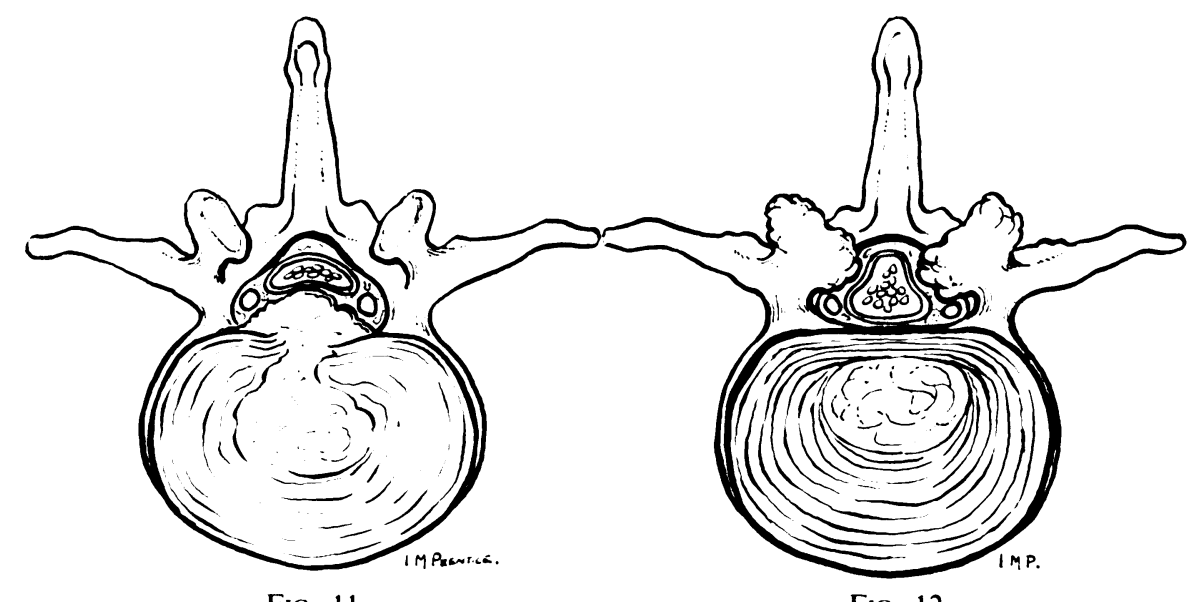

FIG. 11

FiG. 12

Figure 11-Massive central prolapse. Figure 12-Severe hypertrophic osteoarthritis.

Verbiest described clearly the symptoms associated with stenosis-bilateral radicular pains, impairment of sensibility and disturbance of motor power in the legs on standing and walking, but relieved immediately by sitting with the spine flexed.

Myelography may reveal a complete block but it is interesting that the block may be present only with the lumbar spine postured in extension; in flexion the block is relieved (Figs. 7 and 8). This coincides with the characteristic history of symptoms precipitated by walking and standing and relieved by sitting. So marked is this feature in some cases that it has been called "intermittent claudication of the cauda equina" (Blau and Logue 1961). These authors described the paraesthesiae in both feet, spreading upwards on walking and standing and relieved by sitting. Persistence in walking produced increasing weakness of the legs and a sensation of imbalance. 
The term "spinal stenosis" is now more commonly used by clinicians to describe the syndrome, not the congenital narrowing of the canal. Anatomically, stenosis may be primary or secondary.

Primary stenosis-This syndrome associated with a narrow canal is almost entirely confined to men, ranging in age from thirty to seventy years. The symptoms may be precipitated by disc prolapse or degenerative change.

Secondary stenosis-Stenosis in a previously normal canal may be precipitated by massive central prolapse (Fig. 11), severe hypertrophic degenerative change (Fig. 12) or degenerative spondylolisthesis. The clinical features of secondary stenosis resemble those of primary stenosis but they occur in either sex, generally in more elderly patients.

\section{OPERATIVE TECHNIQUE}

Decompression is the essential object of surgical intervention. It must be adequate to give relief of constriction to theca and nerve roots and may involve multiple laminectomy and facetectomy. Stabilisation is a secondary consideration and may be thought necessary in the younger patient if instability is a factor.

\section{LUMBO-SACRAL DISC DEGENERATION}

Lumbo-sacral disc degeneration is a common phenomenon often seen, with degeneration of one or more discs above, during routine radiographic examination. As such it is essentially a radiographic diagnosis and may not cause symptoms. It is a frequent finding in patients under investigation for low back pain, but under these circumstances it is not necessarily the true source of the pain.

There is a syndrome, occurring mostly in women of middle age, which presents as a more definite clinical entity. The patient complains of long-standing low back discomfort and localises the area by drawing the hand across the top of the sacrum. Pain may radiate to both

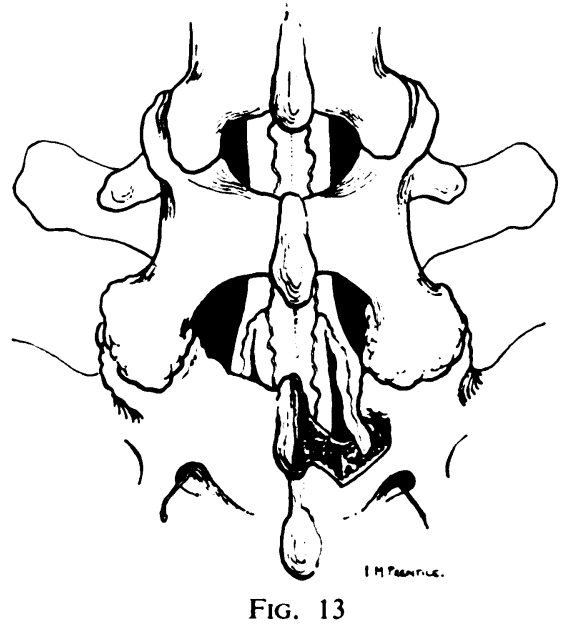

Decompression of a trapped first sacral root. buttocks and the posterior proximal part of both thighs. There may be a definite history of injury such as a heavy fall on the buttocks, or of having hurt the back while lifting a heavy object. Years later the pain arising from osteoarthritis of the lumbo-sacral joints may become apparent.

The picture of this syndrome is occasionally completed by sciatica arising from compression of the first sacral nerve. The nerve is trapped under the medial hypertrophic edge of the superior sacral facet, the anterior component of the lumbo-sacral joint. The nerve may be pushed backwards against this facet by a bulging disc or by a hypertrophic vertebral bar. The spinal canal in the female tends to flatten at the lumbo-sacral level; in the male the canal is narrow more proximally. This factor accounts for the predominance of the syndrome in women.

\section{OPERATIVE TECHNIQUE}

If the low back pain and the pain from first sacral nerve compression become a real burden, unresponsive to conservative measures, the syndrome responds well to a comparatively simple surgical procedure. The site of the true cause of the symptoms is verified by neurological evidence of affection of this nerve.

The lumbo-sacral space is exposed by a midline incision, and clearance of soft-tissue exposes the laminae of the fifth lumbar and first sacral neural arches. Care is taken not to 
weaken the soft tissues controlling the segment above. The ligamentum flavum is excised, exposing the theca and both first sacral roots. One or both are found thickened, white and adherent. The edges of the adjoining laminae are excised and partial vertical facetectomy of the lumbo-sacral joint completes the decompression (Fig. 13). The roots are freed from adhesions and covered with absorbable foam, and posterior fusion of the segment is performed by transarticular screws and posterior laminar grafting.

\section{SUMMARY}

1. Some of the more common and obvious clinical syndromes arising from mechanical and degenerative derangements of the lumbar spine are defined.

2. Some principles in the selection of cases for surgical treatment are discussed and it is stressed how small a part operative intervention plays in the overall problem of low back derangement.

3. Details of surgical technique in the eight types of syndrome are described from past experience in the author's clinic, but not without recognition of the fluidity of this comparatively new field and its continuing evolution.

\section{REFERENCES}

Blau, J. N., and Logue, V. (1961): Intermittent Claudication of the Cauda Equina. Lancet, 1, 1081.

Boucher, H. H. (1959): A Method of Spinal Fusion. Journal of Bone and Joint Surgery, 41-B, 248.

Connolly, R. C., and Newman, P. H. (1971): Lumbar Spondylotomy. Journal of Bone and Joint Surgery, 53-B, 575.

O’Connell, J. E. A. (1951): Protrusions of the Lumbar Intervertebral Discs. Journal of Bone and Joint Surgery, 33-B, 8.

Robinson, R. G. (1965): Massive Protrusions of Lumbar Disks. British Journal of Surgery, 52, 858.

SARPYener, M. A. (1945): Congenital Stricture of the Spinal Canal. Journal of Bone and Joint Surgery, $27,70$. SMITH, L. (1972): Unpublished.

Verbiest, H. (1954): A Radicular Syndrome from Developmental Narrowing of the Lumbar Vertebral Canal. Journal of Bone and Joint Surgery, 36-B, 230.

Verbiest, H. (1955): Further Experiences on the Pathological Influence of a Developmental Narrowness of the Bony Lumbar Vertebral Canal. Journal of Bone and Joint Surgery, 37-B, 576.

Wiltse, L. L., Bateman, J. G., Hutchinson, R. H., and Nelson, W. E. (1968): The Paraspinal Sacrospinalissplitting Approach to the Lumbar Spine. Journal of Bone and Joint Surgery, 50-A, 919. 